\title{
Respective Treatment Modalities with the Use of Combined Ingredients to Address Acne Prone Skin.
}

\author{
Judey Pretorius* \\ Biomedical Emporium, Biomedical Science Research \& Development, South Africa
}

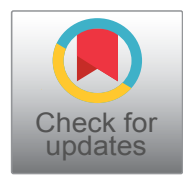

*Corresponding author: Judey Pretorius, Biomedical Emporium, Biomedical Science Research \& Development, Biomedical Emporium, The Willows Office Park, C/O Farm Road and Simon Vermooten, Pretoria, South Africa, 0081, Tel: +27 12809 2856

\section{Introduction}

Acne Vulgaris is a chronic inflammatory condition of the skins' pilosebaceous unit, composed of the hair follicle, arrector pili muscle and the sebaceous gland [1]. Acne typically appears at the high density pilosebaceous units namely the face, neck, upper chest, shoulders and back. 'Acne is the most common dermatologic condition in the world'[2]. Several studies have indicated psychological and emotional distress like social anxiety, poor self-esteem, depression, and suicidal ideation because of acne [2].

\section{Causes}

The causes of acne can differ from one individual to another. The four main interdependent elements are hyperactive sebaceous glands, hyperkeratosis, the bacterium Cutibacterium acnes and the $\mathrm{pH}$ of the skin according to [3].

The sebaceous gland secretes sebum repeatedly. The hair follicle pore distributes the sebum to the skin surface. Hyperactive sebaceous glands over produce sebum which leads to a blockage of hair follicles, resulting in inflammation. At puberty, the increase in testosterone stimulates sebum synthesis, resulting in intensely active sebaceous glands and an increase in acne [3]. Individuals with a more oil prone skin tends to be more acceptable for acne, because of an increase in sebum. The onset of puberty is earlier in Fitzpatrick 5-6 skin types; therefore, acne develop earlier [4]. The luteinizing hormone in females increase after ovulation, which stimulate the sebaceous glands. This causes a rise in acne before menstruation [3].

Hyperkeratosis at the infundibulum of the hair follicle leads to thick, hard material that blocks the hair follicle pore and sebaceous gland resulting in acne papules according to [3]. Cutibacterium acnes (formerly known as Propionibacterium acnes) are commensal species on the skin microbiome. It lives predominantly on the fatty acids in sebum. As the hair infundibulum or follicle gets obstructed by sebum, Cutibacterium acnes multiply. Acne Vulgaris is characterized by the dominance of defined strains of Cutibacterium acnes. Proteins and various digestive enzymes are secreted by Cutibacterium acnes. These enzymes digest sebum and disrupt the cell layers that form the follicles. Cellular destruction, byproducts and bacterial refuse caused by a fast expansion of Cutibacterium acnes in follicles result in inflammation. The bacterium stimulates the infundibulum's epidermal cells, to produce further hyperkeratotic change. Harm to tissue and inflammation caused by Cutibacterium acnes makes the area more receptive to opportunistic bacteria like Staphylococcus aureas [5].

Some research suggest that UV light physically stimulate keratinization and can provoke acne [3]. The use of tobacco products can cause acne according to studies [2]. Inefficient skin hygiene can contribute to the blockage of the hair follicle pores [3].

Sebum hypersecretion and hyperkeratosis (abnormal thickening of the skin outer layer) result in hair pores to narrow and become blocked, this causes a comedo / clogged hair follicle, the first stage of acne. The comedo 
harms the nearby matter which causes inflammation of the tissues near the sebaceous gland opening. The horny material and sebum blocking the hair infundibulum overspill into the dermis. If bacteria occupy the dermis, leukocytes attack the bacteria to form pus. Pus gathers in the dermis and forms an elevated pustule. If the pustule recovers, granulation take place and a scar remains. [3]. It is possible that picking at acne lesions will influence the process of recovery and increase the possibility of scarring [4]. Unevenness, keloids (elevated, visible scars that develop because of an overgrowth of the wound tissue, due to an increase in collagen production), hyper and hypopigmentation are complications that can develop after acne $[6,7]$.

Post inflammatory hyperpigmentation is a common complication of acne, especially in Fitzpatrick 5-6 skin types because of the presence of polymorphonuclear cell infiltrates [4]. The immune response and immunochemical pathway are activated by the population of Cutibacterium acnes in the sebaceous follicle as a noninflammatory comedo develops into inflammatory papules, pustules and nodules. The inflammatory mediators present in the pathway are the cytokines, defensins, peptidases, sebum lipids and neuropeptides [8].

Post inflammatory hyperpigmentation takes place because of an overproduction of melanin pigment (the protein that change the colour of the skin), through the process of melanogenesis (the production of melanin pigment). This proses is initiated by inflammation caused by acne. Inflammation stimulate Propiomelanocortin (POMC) produced by the pituitary gland, the forerunner of the melanin stimulating hormone (MSH). MSH adheres to receptors on the cells in the epidermis namely the keratinocytes and melanocytes. It stimulates the manufacture of the enzyme, Tyrosinase, which are the catalyst of melanin production. L-Dopa and Dopa phosphates are regulators for the melanin granules namely the melanosomes. Melanocytes produce the pigment melanin granules and transport them to the keratinocytes. Keratinocytes absorb and transport the pigment melanin granules throughout the stratum corneum. Melanocytes generate various forms of melanin pigment. Phaemelanin pigment is a melanin pigment that is red in colour and Eumelanin pigment is brown in colour. Eumelanin pigment is higher in Fitzpatric 5-6 skin types, therefore resulting in an increased post- inflammatory hyper pigmentation [9].

The acid mantle is a very thin layer of the uppermost skin surface, on the stratum corneum, which is composed of free fatty acids, sweat and normal skin flora. The acid mantle creates and maintain a somewhat acidic condition ( $\mathrm{pH}$ between 4.5-5.5). This lower $\mathrm{Ph}$ creates a barrier function and sustain a balanced microbiome (the accumulation of all the micro-organisms on the skin tissue). An increase in the skin's Ph will cause a disruption of the microbiome and thus an unfavourable increase of bacteria as Cutibacterium acnes [10].

As an individual enter adulthood, the hormones that play a role in puberty decrease, which leads to a decline in acne. However, it is becoming more prevalent, as research develop, that woman carry acne into maturity. This can be because of hormonal changes due to pregnancy, the menstrual cycle, polycystic ovary syndrome (PCOS), other endocrine disorders and certain medication like corticosteroids, testosterone, and lithium $[6,11]$. Anti-epileptics, anti-cancer drugs (gefitinib) and anabolic steroids [1].

Occlusion or pressure to the skin surface with greasy products and non-hygienic fabric can intensify acne [6]. An example is 'maskne', which is acne developed by wearing a mask to prevent the spread of Covid-19. The mask rest against the skin, the friction can result in sensitivity and inflammation. By enclosing the region with the material, it can possibly produce an unfavourable environment where bacteria, humidity, sweat, sebum, impurities, and heat are entrapped. This can probably influence the skin surface microbiome and $\mathrm{pH}$. The area covered by the fabric presents with acne. The patient's genetic predisposition plays a role, those with an oilier and / or acne prone skin will most likely develop 'maskne'. Those patients who are genetically more prone to a dry or reactive skin, eczema, or dermatitis will most likely develop 'maskitis', when wearing a mask. 'Maskitis' is a form of dermatitis, a general term that describes skin irritation. 'Maskitis' is characterized by small bumps (not acne), redness of a bigger area (not just on a lesion), inflammation, dryness, itchy and flaky skin. 'Maskitis' can appear on the areas of the skin that is not directly covered by the mask. Both these conditions are different, but the root cause is the same. It is highly recommended to wash fabric / cloth masks daily. Disposable masks must be discarded every day.

The face as well as the front part of the mask must not be touched. It must be operated at the regions around the ear or behind the head, this prevents unnecessary contamination [12].

A continuous change in skin care routine can intensify acne by putting stress on the skin and potentially influencing the $\mathrm{pH}$. Studies indicated that staying in an environment with a high humidity can worsen acne. Stress can play a critical role in the activation and aggravation of acne [3]. Increased perspiration leads to a rise in sebum production which can aggravate acne. A family history can also be a contributing factor, studies indicate especially if the mother experienced acne one can experience acne. Environmental conditions as pollution can intensify acne [11].

Early studies indicated that patients with acne had impaired glucose tolerance (insulin resistance) and an 
altered carbohydrate metabolism. A high-Gl diet could strengthen the increase in acne [4].

\section{Types of Acne}

The types of acne are classified as:

1. White heads, called comedones, which are clogged pores, where the head of the pore are closed. It appears as a white or flesh colored speck.

2. Blackheads are also comedones where the clogged pore remains open. The pore is exposed to oxygen which cause the blemish to turn black. Both black and whiteheads are noninflammatory acne.

3. Papules are small, hard, red / pink blemishes with painful sensations, the lesions are inflamed and infected by Propionibacterium acnes $[13,14]$.

4. Pustular acne presents as small white / yellow spots, surrounded by a swollen reddish painful area. It is an infected and inflamed hair follicle that contains pus. Papules and pustules normally occur together in clusters [15].

5. Nodular acne is flesh or red - colored large solid lumps that rest within the dermis. They are painful and seldom points to the surface. Dark marks on the skin may develop above inflamed nodules. Emerging on the face or trunk they may come and go for months [14].

6. Cysts are large painful infected pus-filled lumps, that rest in the dermis. They often burst and infect the surrounding skin. They are the most severe form of acne [13].

\section{Treatments and Products in the Market}

\section{Topical treatments}

Benzoyl Peroxides are an ingredient in overthe-counter topical preparation that works against keratinization, follicular Cutibacterium acnes and inflammation [1].

Topical Retinoids (Vitamin A) are Retinol, Adapalene, Tretinoin and Isotretinoin, they require medical prescriptions. Retinoids act on keratinization and has anti-inflammatory properties. They are contra-indicated in pregnancies. Retinoids induce local reactions and increase the sensitivity of the skin to ultraviolet light. A combination of Topical Retinoids with different mechanisms of actions tend to work more effectively than using a single product [1].

Topical Antibiotics include Clindamycin, Erythromycin and Tetracycline, that work against Cutibacterium acnes and reduce inflammation. These products are often combined with other medication such as topical Retinoids or Benzoyl Peroxide [1].
Salicylic Acid is a chemical exfoliant and an element used in various over-the-counter preparations and prescription medications [1].

Azelaic Acid has antimicrobial and anti-comedonal properties but can cause hypopigmentation in darkerskinned patients [1].

\section{Oral treatments}

Oral Antibiotics include the Tetracyclines like Oxytetracycline, Doxycycline, Lymecycline and Minocycline (Minocycline is not recommended because of side-effects). Aminoglycosides and Chloramphenicol but has limited efficiency [1].

Combined Oral Contraceptives (COC's) contains estrogen and progesterone. Estrogen suppresses the sebaceous glad activity and decrease the formation of ovarian and adrenal androgens. Progesterone only contraceptives worsen acne and should be avoided in woman who have no contraindications against estrogen-containing products [1].

Oral Isotretinoin is normally taken for four to six months, improvement will be noticeable after 8 weeks. If not, retreatment is necessary. It is mostly reserved for severe nodulocystic scarring acne or acne resistant to other therapies. It is teratogenic and must be given with contraception for woman. It can result in side-effects like photosensitivity, dry skin, secondary infection, increased serum lipids and cheilitis [1].

Aloe vera, Pyridoxine, Kampo and Ayurvedic herbal treatments are few complementary and alternative medications (CAM's) that has shown potential benefit, but the studies were inconclusive [1].

\section{Hypothesis}

The correct and effective management of acne still remains challenging in modern day-by-day practice despite the fact that much effort and attention has been directed toward novel technologies and advanced approaches. The scientific rational behind this study is to investigate a more preventative approach for individuals with acne prone skin and to ultimately choose the safest treatment option. The selection of the appropriate treatment is crucial to take the emotional distress of the "patient" into consideration. This study discusses the use of respective products with very specific ingredients by means of case studies in an attempt to describe a simplified and effective approach to manage acne. This study mainly focused on the topical and systemic prevention approach, and to possible factors that play a role on the overall health and ultimately skin health of the "patient".

Oral pharmaceutical ingredients may lead to potential hepatic dysfunction and creates an increased probability of drug reactions. Due to the problems and limitations with the oral administration of scripted 
pharmaceutical compounds, the transdermal route of administration will be focused on, since first-pass metabolism is avoided when utilising the skin for administration, therefore, a wide variety of ingredients can be used topically on the skin which offers more control and less risk.

Several ingredients have been mentioned in the literature to have an effect against acne. These ingredients are also categorised as complementary and alternative medications (CAM's), thus not prescription medicine. The ingredients are incorporated in unique formulations and are presented as products and treatments classified as "cosmeceuticals". It can be denoted that a cosmeceutical is a cosmetic product with medicinal properties.

\section{Methodological Approach and Study Design}

Biomedical Emporium has formulated respective products with familiar ingredients to address the respective concerns of individuals with acne prone skin. Biomedical Emporium products comprise of product available for the public to purchase, as well as professional products available for qualified skin care professionals to perform an aesthetic treatment within a salon or aesthetic clinic environment. These products, such as chemical peels and microneedling (Collagen Induction Therapy) treatments are thus not performed on an individual daily, but rather more sporadically such as monthly treatments. Various home care products and various professional treatments with very specific ingredients were chosen for this study and combined in order to address the various definitional elements of acne, i.e.
a. Elevated levels of sebum production
b. Inflammation
c. Hyperactive keratinization
d. Fluctuating skin $\mathrm{pH}$
e. Microbial growth

Due to the topical and systemic dynamics of this condition, it appears that various ingredients need to be used collaboratively during a treatment protocol for individuals with acne.

The ingredients of choice were;

Niacinamide at $5 \%$

Retinol at $0.1 \%$

Tea Tree Oil at 3\%

Salicylic Acid at 2\% (home care) and Salicylic Acid 20\% (Professional treatment)

Kojic Acid 5\%

Lactic Acid 2\%

Tocopherol Acetate 1\%

\section{Glycolic Acid 25\%}

\section{Zinc Oxide 2\%}

Various home care products and treatments were thus combined together, and a specific treatment protocol was drafted of which an individual had to come for scheduled treatments with a skin professional and use respective home care products.

The products essentially examined are the topical: Anti-Acne Serum, Skin-Repair Serum, and the Enzymatic Therapy Moisturizer.

The professional treatments performed by a skin care professional that were investigated are the: Salicylic Chemical Peel, Glycolic Chemical Peel, Complexion Chemical Peel, Collagen Induction Therapy, i.e., Gold Standard Micro needling (Table 1).

The oral Skin Biotic Collagen Supplement a vitamin rich supplement with hydrolyzed collagen and a probiotic mixed formed part of the systemic treatments.

\section{Product Ingredient Summary}

Topical Retinol (mentioned above) has shown to reduce the formation of acne by preventing hair follicles to clog, this allows treatments and products to work more efficiently. It acts as an exfoliant, by removing keratinized died skin cells. Retinols penetrate towards the dermis, where it stimulates the production of collagen and elastin. It has anti-inflammatory properties $[16,17]$. Retinol is an ingredient that is being used in the Anti-Acne Serum and primarily in the Gold Standard Microneedling treatment.

Tea tree oil has shown to have anti-inflammatory and antimicrobial properties, thus inhibiting the growth of Cutibacterium acnes [18-23]. Tea tree oil is used in the Anti-Acne Serum and the Skin-Repair Serum.

Niacinamide (Vitamin B3) reduces dark spots and scars, redness, and hyperpigmentation. It has an overall brightening effect. Niacinamide strengthens the skin barrier and has anti-inflammatory attributes [24,25]. Niacinamide is used in the Anti-Acne Serum and SkinRepair Serum.

Zinc Oxide represses the production of sebum, has anti-bacterial effect against Cutibacterium acnes and shown anti-inflammatory properties [26-28]. Zinc Oxide is used in the Anti-Acne Serum.

Allantoin has promising wound healing and keratolytic properties as well as skin calming attributes [29]. It is used in both the Anti-Acne Serum and the SkinRepair Serum.

Salicylic Acid, mentioned above, is an Alpha hydroxy acid which is a powerful exfoliant. It removes unwanted material in clogged pores and reduces sebum production. It shed dead keratinized skin cells from the top layers of the skin and works best against mild, 
Table 1: The product and professional treatment ingredient composition.

\begin{tabular}{|c|c|c|c|c|c|c|c|}
\hline $\begin{array}{l}\text { Product or } \\
\text { Professional } \\
\text { treatment: } \\
\text { Ingredients }\end{array}$ & $\begin{array}{l}\text { Anti- Acne } \\
\text { serum }\end{array}$ & $\begin{array}{l}\text { Skin } \\
\text { Repair } \\
\text { serum }\end{array}$ & $\begin{array}{c}\text { Enzymatic } \\
\text { Therapy } \\
\text { Moisturizer }\end{array}$ & $\begin{array}{c}\text { Salicylic } \\
\text { Chemical } \\
\text { Peel }\end{array}$ & $\begin{array}{c}\text { Glycolic } \\
\text { Chemical } \\
\text { Peel }\end{array}$ & $\begin{array}{l}\text { Complexion } \\
\text { Chemical Peel }\end{array}$ & $\begin{array}{l}\text { Gold standard } \\
\text { Microneedling }\end{array}$ \\
\hline Product & $x$ & $X$ & $x$ & & & & \\
\hline $\begin{array}{l}\text { Professional } \\
\text { treatment }\end{array}$ & & & & $\mathrm{X}$ & $x$ & $\mathrm{X}$ & $\mathrm{X}$ \\
\hline Retinol & $x$ & & & & & & $x$ \\
\hline Tea tree oil & $x$ & $X$ & & & & & \\
\hline $\begin{array}{l}\text { Niacinamide } \\
\text { (Vitamin B3) }\end{array}$ & $x$ & $X$ & & & & & \\
\hline Zinc Oxide & $x$ & & & & & & \\
\hline Allantoin & $x$ & $X$ & & & & & \\
\hline $\begin{array}{l}\text { Salicylic } \\
\text { Acid }\end{array}$ & & $x$ & & & & & \\
\hline $\begin{array}{l}\text { Alpha- } \\
\text { Tocopherol }\end{array}$ & & $X$ & & & & & \\
\hline $\begin{array}{l}\text { Keratolic } \\
\text { enzymes }\end{array}$ & & & $x$ & & & & \\
\hline Shea Butter & & & $x$ & & & & \\
\hline $\begin{array}{l}\text { Salicylic } \\
\text { Acid }\end{array}$ & & & & $x$ & & $x$ & \\
\hline $\begin{array}{l}\text { Glycolic } \\
\text { Acid }\end{array}$ & & & & & $x$ & $x$ & \\
\hline Lactic Acid & & & & & & $X$ & \\
\hline Kojic Acid & & & & & & $x$ & \\
\hline
\end{tabular}

comedonal black and whiteheads [20]. Salicylic acid is an ingredient used in the Skin Repair Serum.

Alpha-Tocopherol (Vitamin E) used in the Skin Repair Serum, is a natural antioxidant occurring in healthy skin, which tend to get depleted over time. It improves cell regeneration and protects the skin against environmental stressors like pollution. It reduces UV damage and has anti-inflammatory attributes. It shown fast absorbing and fast healing properties $[22,30]$.

The Enzymatic Therapy treatment is composed of keratolytic enzymes which has exfoliant properties. It dissolves the protein bonds between dead keratinized cells in acne. It prevents environmental damage. The shea butter in this product contains vitamins and essential fatty acids. It assists with UV protection and collagen production. The Vitamin E in the Shea butter has anti-inflammatory, antioxidant, and regeneration properties. It increases the healing rate of acne lesions and reduces redness. It corrects skin discoloration and assist with an even skin tone [31].

The Skin Biotic Collagen Supplement taken orally improves the systemic health and gut flora of the digestive system, through its probiotic contents. It is a source of multi vitamins and minerals. It contains ingredients like Vitamin C and Vitamin B3 that reduce Vitamin B3 deficiencies, support cellular energy production, brain function, a healthy metabolism and liver function. Himalayan crystal salts are the purest salt form, it regulates blood sugar levels, hormonal imbalances and improves insulin sensitivity [32]. Folic acid, produce and maintain new cells and prevent changes to the DNA [33]. Biotin, regulates blood sugar levels, protect the nervous system, skin, and hair health [34]. By taking the supplement it reduces the severity of skin sensitivities and allergies. It assists with a balanced lifestyle and improves the overall skin health.

The supplement can be diluted with Micro-Clustered Water. It has a high oxygen level, which assists with energy production, glycolysis, alcohol metabolism and the reduction of toxins. It has a high $\mathrm{Ph}$, which enables improved blood flow, thus increases oxygen delivery [35].

\section{Non-prescribed Professional Skin Care Treatments}

Treatments received at aesthetic practices such as salons formed part of the investigation.

Chemical Peels create careful exfoliation by using 
acids. It lowers the skin $\mathrm{pH}$ and loosen the protein bonds between the dead keratinized skin cells. This promotes growth of healthy skin cells. Different depths and strengths are available [16]. Chemical Peels work on the outer layer (epidermis) and deeper layer (dermis) of the skin depending on the strength. A decreased amount of material will clog the pores resulting in a decline of acne after a few applications in a 4-6-week cycle [36].

The Salicylic Chemical Peel composes of Salicylic acid as main ingredient, mention above.

The 25\%Glycolic Acid Chemical Peel consist of Glycolic acid. An Alpha hydroxy acid which is a chemical exfoliant with antioxidant activity that stimulate collagen growth [26].

The Complexion Chemical Peel is a stronger Chemical Peel which penetrates deeper. It contains Glycolic acid, Lactic acid, Salicylic acid and Kojic acid. Lactic acid is an Alpha hydroxy acid, another chemical exfoliant that assist with the reduction of acne lesions, scars, and pigmentation. It improves uneven skin tone [37]. Kojic acid is isolated from a fungus and is a byproduct of certain fermented foods. It is a Chemical exfoliant that inhibits the formation of the amino acid, tyrosine, which reduces melanin production. It acts as a skin lightener. It has antioxidant, anti-bacterial and antifungal properties [38].

Collagen Induction Therapy is an electronic technique where, multiple sterile needles are used to rupture the skin to a pre-establish depth to create a "controlled" wound. The body will react and transmit collagen and elastin to restore the entire area. It stimulates the dermis to prompt cell turnover and produce healing factors [19]. This improves the formation of acne, acne complications and the appearance of large pores. Collagen Induction Therapy can be performed with the Gold Standard Microneedling. It enables the ingredients to penetrate the skin. Gold Standard Microneedling is a Collagen Induction Therapy procedure performed with Retinol as main ingredient. Instead of placing Retinol on the skin it will enable Retinol to infiltrate into the deeper layers of the skin and work more effectively [39]. This treatment can be done in 4-6-week intervals in alteration with a Chemical Peel procedure only.

\section{Study Design and Clinical Cases}

Individuals from all walks of life, with different severities and types of acne as well as genetic predispositions were identified. The treatment approach for the study was to use a combination of BioMedical Emporium's diverse products and treatments. This formed part of a holistic, well balanced preventative strategy. The treatment improvement of acne and complications were evaluated monthly. Skin care professionals performing the treatments, confirmed the products used at home and the treatment protocol. Questions were asked regarding the patients' acne occurrence, expression, and lifestyle. Blood and urine laboratory tests were available if the subject willfully agreed to participate. All laboratory testing of the respective subject's blood and urine was performed by either a specialist Chemical Pathologist or a registered nurse at an accredited laboratory under the national standards and legislation of the Republic of South Africa. The laboratory test results were used as a method to detect systemic imbalances which could play a contributing role in acne.

The ethical principles for this research were aligned with the declaration of Helsinki. The primary purpose of this research study involving human subjects is to understand the causes, development and effects of acne and improve preventive, diagnostic and therapeutic interventions for safety, effectiveness, efficiency, accessibility, and quality. All subjects willfully participated, and signed an indemnity prior to the acquisition of products and formulated professional treatment protocols.

All professional skin treatments were conducted by individuals with a diploma in advanced dermal aesthetics. All the professional treatments that were performed was done in a controlled environment at the respective aesthetic facility. The respective subjects consciously participated in the study and completed an indemnity form prior to the aesthetic treatment performed. None of the treatments that were performed were invasive or uncomfortable. In fact, the whole purpose of this study was to create an environment of harmony and peace to address a very stressful concern for individual's with acne. Thus, the risk was profoundly reduced by rather creating a favourable and safe environment for the subject to address the concern of acne, before the subject is exposed to more invasive treatments and or prescribed medication. Subject were requested to complete a client form requesting to divulge all relevant medical history, allergy or pregnancies.

The skin care professional also had to be conscious of the subject's budget. Although, the ideal would be to perform more frequent treatments on the various subject's, this was not always the case and a home care plane and professional treatment plan had to carefully be outline to the subjects to accommodate their budget as well as to ensure that a favourable result be obtained.

\section{Case Study Discussion}

The study included 6 individuals. 5 Females and one male. The average age between participants were between $16-25$ years.

\section{Subject 1}

Summary: Female, 16 years presented with severe active acne (popular, pustular, nodular, and cystic) on the entire face. Puberty, emotional stress, and an active lifestyle with increased perspiration can be the theoretical contributing factors. 


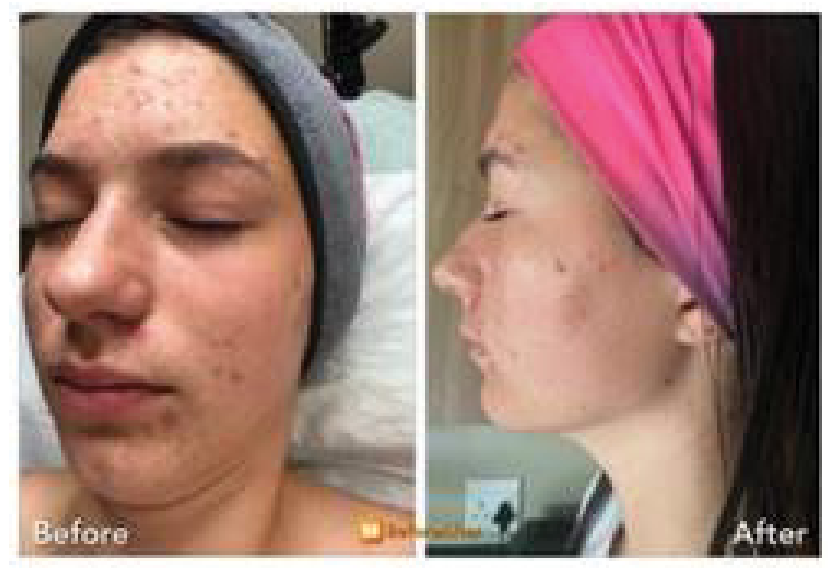

Figure 1: Female, 16 years presented with severe active acne (popular, pustular, nodular, and cystic) on the entire face. Puberty, emotional stress, and an active lifestyle with increased perspiration can be the theoretical contributing factors.
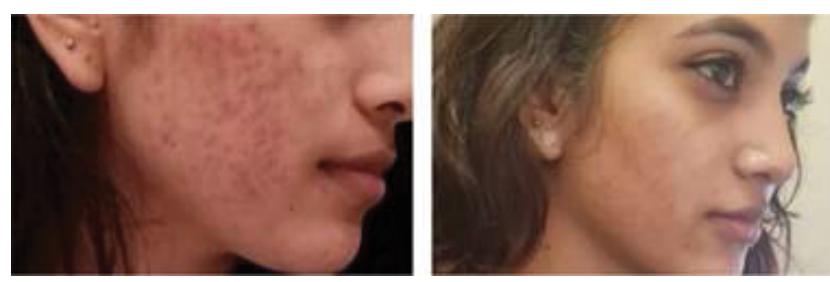

Figure 2: Female, 23 years with moderate to severe acne (comedonal, popular and pustular) with noticeable dark acne lesions and hyperpigmentation, experienced on the cheek area. Subject 2, an adolescent has experienced hormonal imbalances after puberty. Subject 2, presented with an "oil skin type" which constituted to an increased sebum production. Subject 2, is also located in an environment with high humidity over $70 \%$.
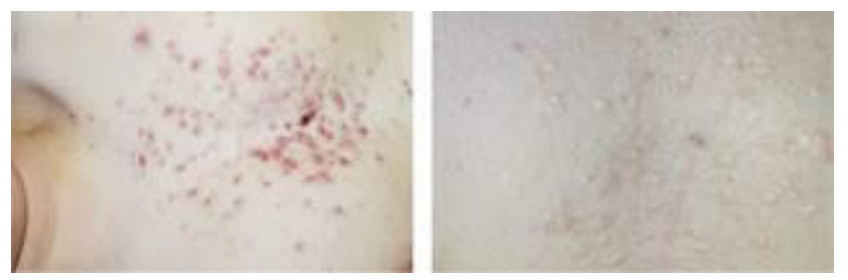

Figure 3: Male, 19 presented with severe acne (combination of comedonal, popular and pustular) with visible inflammation and infection on the chest area. Over time, the scaring of the acne, due to picking converted into keloid and hypertrophic scars. Hormonal imbalances due to puberty and emotional stress could be theoretical contributing factors. Subject 3 also indicated that he was using respective prescribed medication for anxiety and depression.

Treatment protocol: The patient received the $25 \%$ Glycolic Chemical Peel and the 20\% Salicylic Acid Chemical Peel with one month apart between each treatment. For home care, Subject 1 used the Facial Cleanser, Anti-Acne Serum, Enzymatic Therapy, and the Skin Biotic Supplement as home care products (Figure 1).

Outlined in Table 2 below is the home care treatment regime of Subject 1
Table 2: Home skin care routine of Subject 1.

\begin{tabular}{|l|c|c|}
\hline & Morning (AM) & Evening (PM) \\
\hline Facial Cleanser & $\mathrm{X}$ & $\mathrm{X}$ \\
\hline Anti-Acne Serum & & $\mathrm{X}$ \\
\hline Enzymatic Therapy & $\mathrm{X}$ & $\mathrm{X}$ \\
\hline
\end{tabular}

Table 3: Home skin care routine of Subject 3.

\begin{tabular}{|l|c|c|}
\hline & Morning (AM) & Evening (PM) \\
\hline Anti-Acne Serum & & $\mathrm{X}$ \\
\hline Skin Repair Serum & $\mathrm{X}$ & \\
\hline Enzymatic Therapy & $\mathrm{X}$ & $\mathrm{X}$ \\
\hline
\end{tabular}

\section{Subject 2}

Summary: Female, 23 years with moderate to severe acne (comedonal, popular and pustular) with noticeable dark acne lesions and hyperpigmentation, experienced on the cheek area. Subject 2, an adolescent has experienced hormonal imbalances after puberty. Subject 2, presented with an "oil skin type" which constituted to an increased sebum production. Subject 2 , is also located in an environment with high humidity over $70 \%$.

Treatment protocol: Subject 2, received two Gold Standard Microneedling treatments with a month apart between treatments (Figure 2).

\section{Subject 3}

Summary: Male, 19 presented with severe acne (combination of comedonal, popular and pustular) with visible inflammation and infection on the chest area. Over time, the scaring of the acne, due to picking converted into keloid and hypertrophic scars. Hormonal imbalances due to puberty and emotional stress could be be theoretical contributing factors. Subject 3 also indicated that he was using respective prescribed medication for anxiety and depression. The Subject was also prescribed oral Isotretinoin by his doctor to prevent new acne from forming.

Treatment protocol: The patient received two chemical peel treatments with a combination of ingredients of $25 \%$ Glycolic Acid Peel and $20 \%$ Salicylic Acid Peel (the peels where layered), once per month for three months after the keloids formed. The subject's skin care routine consisted of the Anti-Acne Serum, the Skin-Repair Serum as well as the Enzymatic Therapy Moisturizer (Figure 3 and Table 3).

\section{Subject 4}

Summary: Female, 52 severe acne (comedonal, popular, pustular, and nodular) which appears in phases was experienced on the entire face. The patient is a diabetic patient with an impaired glucose tolerance and an insulin deficiency. The patient is on blood pressure, cholesterol, and diabetic type two medication. She previously took oral Isotretinoin, but 

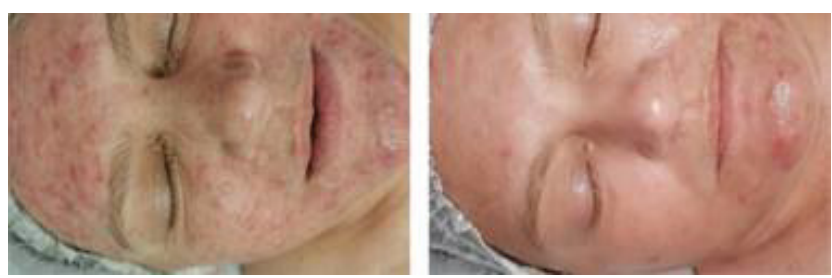

Figure 4: Female, 52 severe acne (comedonal, popular, pustular, and nodular) which appears in phases was experienced on the entire face. The patient is a diabetic patient with an impaired glucose tolerance and an insulin deficiency. The patient is on blood pressure, cholesterol, and diabetic type two medication.
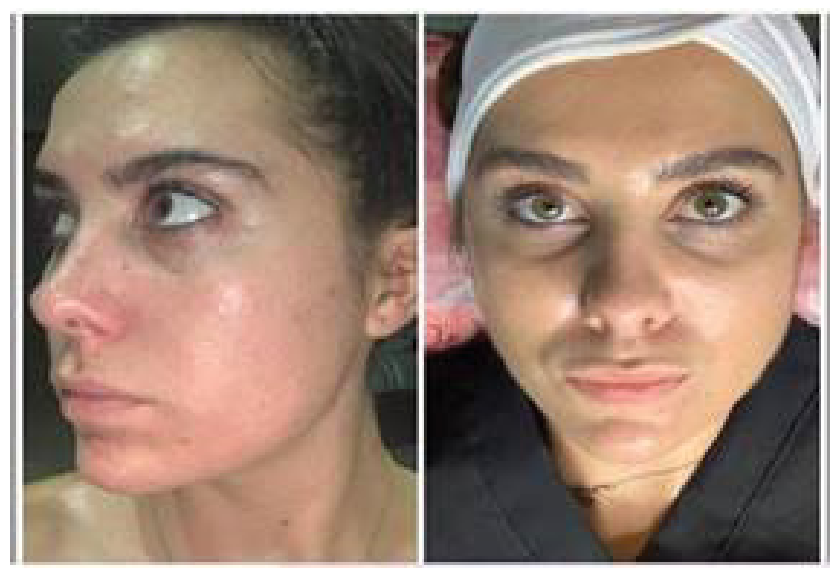

Figure 5: Female, 25 mild acne (comedonal acne with popular lessions) on the face, especially during the menstrual cycle. The patient, an adolescent has suffered with acne since puberty, due to hormonal imbalances. The subject presented with a sensitive, oil, and acne-prone skin.

the acne reappeared and increased after six months. The oral Antibiotic, Lymecycline, was prescribed but was not effective. The patient performed clinical laboratory blood tests and the following markers were detected. The patients s-g-Glutamyl transferase and the S-ALT enzymes were high which indicated that the liver, was compromised which can be devoted to the use of medication and smoking tobacco. A destructive liver result in hormonal imbalances which can contribute to acne.

Treatment protocol: The patient received six Glycolic Chemical Peel treatments every two weeks. The products that she used were the Skin Repair Serum, Enzymatic Therapy, and the oral Skin Biotic Supplement (Figure 4 and Table 4).

\section{Subject 5}

Summary: Female, 25 mild acne (comedonal acne with popular lessions) on the face, especially during the menstrual cycle. The patient, an adolescent has suffered with acne since puberty, due to hormonal imbalances. The subject presented with a sensitive, oil, and acneprone skin. The multiple changes in her skin care routine with various products available on the market, as an attempt to find the best cosmetic product, only
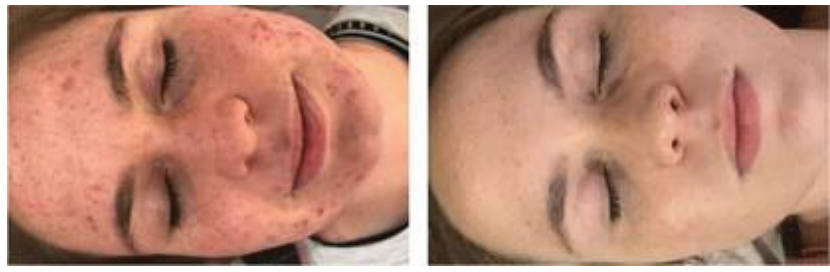

Figure 6: Female, 20, severe acne (pupular, pustular, nodular, and cystic) on the entire face. The subject started experiencing acne since puberty, at the age of 17 up until early adolescence.

Table 4: Home skin care routine of Subject 4.

\begin{tabular}{|l|c|c|}
\hline & Morning (AM) & Evening (PM) \\
\hline Skin Repair Serum & $\mathrm{X}$ & $\mathrm{X}$ \\
\hline Enzymatic Therapy & $\mathrm{X}$ & $\mathrm{X}$ \\
\hline Skin Biotic Supplement & $\mathrm{X}$ & \\
\hline
\end{tabular}

Table 5: Home skin care routine of Subject 5.

\begin{tabular}{|l|c|c|}
\hline & Morning (AM) & Evening (PM) \\
\hline Skin Repair Serum & $\mathrm{X}$ & $\mathrm{X}$ \\
\hline Enzymatic Therapy & $\mathrm{X}$ & $\mathrm{X}$ \\
\hline
\end{tabular}

Table 6: Home skin care routine of Subject 6.

\begin{tabular}{|l|c|c|}
\hline & Morning (AM) & Evening (PM) \\
\hline Anti-Acne Serum & & $\mathrm{X}$ \\
\hline Skin Repair Serum & $\mathrm{X}$ & \\
\hline Enzymatic Therapy & $\mathrm{X}$ & $\mathrm{X}$ \\
\hline
\end{tabular}

amplified the acne.

Treatment protocol: The subject received two $25 \%$ Glycolic Peel treatments every six weeks. The Skin Repair Serum and the Enzymatic Therapy formed part of her skin care routine (Figure 5 and Table 5).

\section{Subject 6}

Summary: Female, 20, severe acne (pupular, pustular, nodular, and cystic) on the entire face. The subject started experiencing acne since puberty, at the age of 17 up until early adolescence. Three months before the subject experienced severe acne she started with oral Isotretinoin, which according to the subject only aggravated her acne even more. The subject is a Type 1 diabetic, therefore has an altered glucose metabolism which could be a contributing factor.

Treatment protocol: The patient received 20\% Salicylic Acid Chemical Peel every 4 weeks for a year. The subject used the Anti-Acne Serum, the Skin Repair Serum, and the Enzymatic Therapy Moisturizer as home care. The treatment improves the patient's overall appearance and skin health at a young age (Figure 6 and Table 6).

\section{Concluding Remarks}

The combined use of ingredients such as Retinol, 
Tea Tree Oil, Niacinamide, Zinc Oxide, Allantoin, Alpha-Tocopherol, Shea Butter, Salicylic Acid, and other Keratolytic Enzymes has indicated a therapeutic effect against acne. These ingredients in studied predetermined combinations, with the correct additives, formulated according to proven manufacturing procedures showed promising results. Acids such as Salicylic Acid, Glycolic Acid, Lactic Acid and Kojic Acid in Chemical Peel treatments and in combination with Collagen Induction Therapy (microneedling), plays a fundamental role in the prevention and treatment of acne and acne complications.

The laboratory blood test results indicated that taking medication may have an influence on hepatic dysfunction and ultimately effect overall skin health. Hormonal imbalances and the use of prescribed medication may most certainly constitute to systemic fluctuations which inherently may cause the expression of acne on the skin. Therefore, some emphasis on the importance of following the complementary and alternative medicine approach (CAM's) is a plausible route for an acne treatment protocol. This option minimises the risk in the prevention and treatment of acne. The safest approach is also mainly the preferred option when treating children and teenagers. In some instances, teenagers are not yet concerned and affected by the acne. Therefore, the complementary and alternative medicine approach would be most recommended. This would serve as a foundation to teach the child or teenager the importance of following a high-quality home care routine. The laboratory tests also indicate the importance of an individual's overall health. Striving to follow a healthy lifestyle contributes to a healthy skin. This includes consuming a low-GI diet, thus maintaining a healthy glucose metabolism, exercise, eliminating tobacco products and alcohol and reduce stress. Combining the topical route, products and treatments, with the systemic route by adhering to a healthy lifestyle will result in a holistic, more conservative approach. This accentuates the importance of prevention. In some cases, combing the therapy, with schedule medication as the oral contraceptive or Isotretinoin with a Medical Doctors recommendation, can produce synergistic results.

This alternative safe integrative approach towards the prevention and treatment of acne and acne complications contribute to the uniqueness of this study. This approach is ideal for patients and younger individuals that do not want to undergo intensive treatments as the use of prescribed topical and systemic medicine to treat acne. Systemic markers that are contributing factors towards acne are detected in clinical blood laboratory tests, these markers can be altered by changing certain lifestyle habits.

The holistic, preventative approach that includes: a good skin care routine, the use of the correct home care products, receiving the appropriate, timely treatments with a skin care professional, and adhering to a healthy lifestyle plays a fundamental role in the struggle against acne. Further studies towards complementary and alternative medicine (CAM's) in 'Cosmeceutical' formulations for acne prevention and treatment will be advantageous. A correlation between systemic contributing factors and the presence of acne must be further investigated $[40,41]$.

\section{Acknowledgements}

Respective aesthetic practices across South Africa are certified stockists of Biomedical Emporium products. These stockists are all formally qualified within the discipline of dermal aesthetics. They have participated in this study.

1. Bonheur (Cape Town, Western Cape, RSA)

2. Brow Architect (Johannesburg, Gauteng, RSA)

3. Cherish (Westville, KZN, South Africa)

4. Dermastetix Skin Care Studio (George, Western Cape, RSA))

5. Sabella (Brits, North West, RSA)

6. Salon Antoinie (Potchefstroom, North West, RSA)

\section{References}

1. Williams HC, Dellavalle RP, Garner S (2011) Acne vulgaris. The Lancet 379: 361-372.

2. Dréno B, Araviiskaia E, Kerob D, Andriessen A, Anfilova M, et al. (2020) Nonprescription acne vulgaris treatments: Their role in our treatment armamentarium- An international panel discussion. Journal of Cosmetic Dermatology 19: 2201-2211.

3. Mitsui T (1997) New Cosmetic Science. (1 ${ }^{\text {st }}$ edn), Elsevier Science B.V, Amsterdam, The Netherlands.

4. Bhate K (2012) Epidemiology of acne vulgaris. British Journal of Dermatology 474-485.

5. Wikipedia, The Free Encyclopedia (2021) Cutibacterium acnes.

6. Mayo Clinic (2020). Acne.

7. Harvard Health Publishing (2019) Keloids.

8. Tanghetti EA (2013) The Role of Inflammation in the Pathology of Acne. J Clin Aesthet Dermatol 6: 27-35.

9. Milward A (2017) Post inflammatory hyperpigmentation -understanding melanogenesis. Medico Beauty Institute.

10. Searcy B (2020) Impact of pH on Acne. Rain Organica.

11. Palmer A (2020) An overview of acne vulgaris. Verywellhealth.

12. Gross D (2020) Gives tips to identify maskne vs. maskitis. Cision PR Newswire.

13. Thomas L (2019) Papules, pustules, and nodules. News medical Life Science.

14. Collier $L$ (2020) Types of acne pimples. Healthgrades.

15. Freeland $M(2019)$ The 6 types of acne and how to treat them. GoodRx. 
16. Perkins S (2020) How do chemical peels work? Medical News Today.

17. Perkins S (2020) Can retinol be used to Treat Acne? Healthline.

18. Cobb C (2019) Everything you need to know about lactic acid peels.

19. Cobb C (2019) What is microneedling? Benefits and use. Medical News Today.

20. Cobb C (2019) Can salicylic acid help treat acne? Healthline.

21. Cobb C (2019) How does tea tree oil help the skin? Healthline.

22. Cobb C (2019) Is vitamin e helpful or harmful for treating acne? Healthline.

23. Cobb C (2019) Salicylic Acid vs. Benzoyl Peroxide: Which is better for acne? Healthline.

24. Barr K (2020) Vitamin B3 in skin care: 5 Benefits to using a niacinamide serum.

25. Miller A (2021) Six ways you can benefit from supplementing with nicotinamide riboside (NR). Thorne.

26. Cobb C (2020) Is glycolic acid a good acne treatment? Healthline

27. Cobb C (2020) What is radiofrequency skin tightening? Healthline

28. Cobb C (2020) Zinc for acne: Does it work? Medical News Today.
29. Tamatam S (2020) Allantoin: A safe \& effective skin care ingredient you need to know about. Skinkraft.

30. Vitagene (2021) Why vitamin E can impact acne and skin health.

31. Vitagene (2021) Why you should consider shea butter for acne and skin.

32. NDTV.FOOD (2017) 6 Wonderful Benefits of Himalayan Salt: The Purest Salt on Earth.

33. Drugs.com (2021). Folic acid.

34. Wilson D (2019) Health Benefits of Biotin. Healthline.

35. Stanton B (2021) O2 Blog: Does oxygenated water have health benefits? Drink $\mathrm{O} 2$.

36. Arizonadermatology (2021). How do chemical peels work? What can they treat?.

37. Seladi-Schulman J (2017) Kojic acid: What you need to know. Medical News Today.

38. Bhattacharya S (2020) Kojic acid: Your ultimate solution to skin pigmentation. Skinkraft.

39. Friedman $R$ (2018) The biomedical emporium gold standard retinol needling treatment. Aesthetic \& Anti-Ageing.

40. Dermalux (2021) The most advanced portable LED system in the world.

41. Harvard Health Publishing (2019) LED lights: Are they a cure for your skin woes? 